\title{
ANÁLISE ESTATÍSTICA E OPTIMIZAÇÃO DE PERFIS DE REDUÇÃO TERMOPROGRAMADA (TPR)
}

Marcio Nele*

Escola de Química, Universidade Federal de Rio de Janeiro, Bloco E, Ilha do Fundão, 21949-900 Rio de Janeiro - RJ, Brasil

Esteban Lopez Moreno

Centro de Tecnologia Mineral, Ilha do Fundão, 21949-900 Rio de Janeiro - RJ, Brasil

Heloysa Martins Carvalho Andrade

Instituto de Química, Universidade Federal da Bahia, Campus de Ondina, 40170-290 Salvador - BA, Brasil

Recebido em 11/2/05; aceito em 14/10/05; publicado na web em 14/3/06

\begin{abstract}
STATISTICAL ANALYSIS AND OPTIMIZATION OF TEMPERATURE PROGRAMMED REDUCTION (TPR) ANALYSIS. The effect of operational variables and their interaction in TPR profiles was studied using a fractional factorial experimental design. The heating rate and the reducing agent concentration were found to be the most important variables determining the resolution and sensitivity of the technique. They showed opposite effects. Therefore, they should be manipulated preferentially in order to obtain optimized TPR profiles. The effect of sample particle size was also investigated. The tests were carried out within a $\mathrm{Cu} / \mathrm{Zn} /$ $\mathrm{Al}_{2} \mathrm{O}_{3}$ catalyst used for the water-gas shift reaction that presented two distinct species of $\mathrm{Cu}^{2+}$ in TPR profiles.
\end{abstract}

Keywords: TPR; temperature programmed reduction; experimental design.

\section{INTRODUÇÃO}

A redução termoprogramada (TPR) é uma técnica de caracterização de materiais que faz parte do conjunto de técnicas conhecidas como termoanalíticas. O perfil de TPR é capaz de fornecer uma impressão digital do material analisado e prover informações sobre a estrutura interna e superficial, a um baixo custo operacional e de montagem do equipamento ${ }^{1}$. Para catálise heterogênea esta técnica apresenta as vantagens adicionais de permitir o estudo da superfície do catalisador em condições próximas às reacionais, sem necessidade de vácuo.

A idéia básica da técnica, pioneiramente utilizada por Robertson $^{2}$ em um equipamento similar ao desenvolvido por Cvetanovic $^{3}$ para estudos de TPD (dessorção termoprogramada), consiste em monitorar a redução de uma amostra sob fluxo de gás redutor (usualmente $\mathrm{H}_{2}$ ou CO) diluído em gás inerte $\left(\mathrm{N}_{2}\right.$ ou $\mathrm{Ar}$ ), enquanto a temperatura é aumentada linearmente com o tempo. A técnica é altamente sensível à presença de espécies redutíveis e tem sido muito utilizada na caracterização de catalisadores mássicos e suportados, especialmente em problemas como estudo da redução de íons em zeólitas ${ }^{4}$, influência de diferentes suportes em um sistema catalítico (SMSI - "Strong Metal-Support Interaction") ${ }^{5-7}$, influência das condições dos pré-tratamentos nas propriedades dos catalisadores $^{6,8}$, efeito dos íons dos metais de transição na redutibilidade de óxidos metálicos ${ }^{9}$, investigação de formação de ligas em catalisadores multimetálicos e o estado do metal no catalisador ${ }^{1}$.

Apesar da grande importância da interpretação dos perfis de redução em relação às variáveis operacionais (concentração de agente redutor, vazão de gás, taxa de aquecimento e quantidade de amostra), poucos são os trabalhos na literatura que lidam com a influência das condições experimentais nos perfis de redução ${ }^{10}$.

Bosh $^{11}$ pesquisou a influência das variáveis operacionais nos perfis de redução do $\mathrm{V}_{2} \mathrm{O}_{5}$ e Monti ${ }^{12}$ analisou a influência dessas variáveis operacionais sobre a temperatura de máximo consumo

*e-mail: nele@eq.ufrj.br de $\mathrm{H}_{2}(\mathrm{Tm})$ na redução do NiO. Esses autores observaram tendências nos efeitos das variáveis operacionais, como o aumento da temperatura de máxima redução com o aumento da taxa de aquecimento, e propuseram um critério, baseado no consumo máximo e mínimo de hidrogênio, para escolha adequada das condições experimentais em TPR.

O efeito causado pela variação dos parâmetros operacionais na temperatura de máximo consumo de hidrogênio na redução de sistema cobre-zeólita foi estudado por Gentry ${ }^{4}$, que propôs um método para estimativa das constantes cinéticas sem necessidade de integração das equações diferencias. Este método, no entanto, somente é aplicável a cinéticas de reação lineares. Outras cinéticas típicas de reações heterogêneas foram estudadas por $\operatorname{Lin}^{13}$, que demonstrou que a cinética linear pode mascarar cinéticas mais complexas, levando o investigador a acreditar erroneamente que o sistema estudado pode ser tratado realmente com cinética de primeira ordem.

Fierro $^{14}$ pesquisou a sensibilidade dinâmica dos perfis de redução, através de simulação, em relação às variáveis operacionais. Neste sistema a taxa de reação observada foi de ordem zero em relação ao $\mathrm{H}_{2}$, e o perfil observado é resultado da adsorção-dessorção ativada de $\mathrm{H}_{2}$ simultaneamente ao consumo.

Kislyuk $^{15}$ investigou, através de simulações, o efeito das interações laterais entre as espécies adsorvidas no catalisador e concluiu que este efeito aumenta a energia de ativação estimada experimentalmente. Tonge ${ }^{16}$ estudou a influência da distribuição de diâmetros de partícula da amostra e sugeriu que a faixa de diâmetro de partícula deve ser o mais estreita possível para prevenir o aparecimento de artefatos no perfil de redução.

O conhecimento da influência das variáveis experimentais na posição do pico de redução, na forma do perfil de redução, na sensibilidade da técnica e na resolução entre diferentes espécies é essencial para a escolha correta das condições operacionais. Este trabalho tem como objetivo investigar quantitativamente a influência das variáveis operacionais nestes parâmetros, usando planejamento estatístico de experimentos. Através da análise quantitativa destes efeitos, é possível hierarquizar a importância das variáveis e 
escolher adequadamente as condições experimentais nas quais os experimentos deverão ser executados.

\section{PARTE EXPERIMENTAL}

Para estudar o processo de redução do catalisador $\mathrm{CuO} / \mathrm{ZnO} /$ $\mathrm{Al}_{2} \mathrm{O}_{3}$, escolheu-se uma amostra de razão atômica $\mathrm{Cu} / \mathrm{Zn} / \mathrm{Al}=40$ / $45 / 15$ altamente ativa para a reação de $\operatorname{shift}^{17}$. Esta amostra foi preparada pela OXITENO S.A., segundo o método desenvolvido por Lima ${ }^{17}$. As sínteses foram realizadas através da técnica de coprecipitação, adicionando-se uma solução dos nitratos metáli$\cos \left(2,7 \mathrm{~mol} \mathrm{~L}^{-1}\right)$ a uma solução de carbonato de sódio $\left(0,13 \mathrm{~mol} \mathrm{~L}^{-1}\right)$,

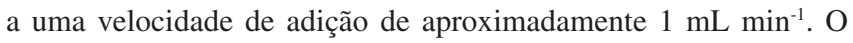
precursor assim obtido foi maturado por $6 \mathrm{~h}$ em $\mathrm{pH} 5$, lavado exaustivamente com água deionizada, seco em estufa a $160{ }^{\circ} \mathrm{C}$ por $4 \mathrm{~h}$ e calcinado a $350{ }^{\circ} \mathrm{C}$ por $3 \mathrm{~h}$. Após estas etapas, o catalisador foi moído em almofariz e peneirado na granulometria desejada.

O sistema utilizado para análises de TPR foi montado no próprio laboratório. Em um experimento típico, uma amostra com o diâmetro de partícula entre 40 a $100 \mu \mathrm{m}$ era colocada em reator de leito fixo em um forno, submetida à taxa de aquecimento controlada sob fluxo constante $\left(\mathrm{F}=30 \mathrm{~cm}^{3} \mathrm{~min}^{-1}\right)$ de mistura de $\mathrm{H}_{2} / \mathrm{N}_{2}$ de concentração 3 ou $5 \%$. As medidas de redução foram feitas após a passagem do gás redutor à temperatura ambiente por um período de aproximadamente $10 \mathrm{~min}$. Os gases efluentes eram analisados em fluxo contínuo usando um cromatógrafo (Instrumentos Científicos CG Ltda.) com detector de condutividade térmica (DCT).

Os principais parâmetros de uma técnica analítica são capacidade de identificação das espécies presentes na amostra, resolução entre diferentes espécies e sensibilidade a estas em concentrações reduzidas. Para avaliar estes fatores no sistema $\mathrm{CuO} / \mathrm{ZnO} / \mathrm{Al}_{2} \mathrm{O}_{3}$, as respostas analisadas foram a temperatura de máximo consumo de $\mathrm{H}_{2}$ da primeira espécie de $\mathrm{CuO}\left(\mathrm{Tm}_{1}\right)$, a resolução avaliada pela distância entre os dois picos principais (Res - Equação 1) e a sensibilidade (Sens - Equação 2).

$\operatorname{Res}=\frac{T m_{2}-T m_{1}}{\beta}$

Sens $=\frac{C\left(T=T m_{1}\right)}{C o}$

onde, $T m_{2}$ e $T m_{1}$ são a temperatura de máximo consumo da primeira e segunda espécies de $\mathrm{Cu}^{2+}, \beta$ a taxa de aquecimento $\left(4{ }^{\circ} \mathrm{C}\right.$ $\left.\min ^{-1}\right)$, Co a concentração original de hidrogênio $(2,72 \mathrm{mmol} \mathrm{cm}$ ${ }^{3}$ ) e $C$ o consumo do agente redutor, hidrogênio, avaliado na temperatura $T m_{l}$.

Em estudos de redução termoprogramada, é necessário corrigir os atrasos relativos à medida da temperatura e o registro do consumo de gás, ou pode-se incorrer em erros graves nos parâmetros cinéticos obtidos ${ }^{18}$. Assim:

$$
T=T_{\text {med }}+\beta\left(\tau-\frac{V_{t}}{F}\right)
$$

onde $T$ : temperatura corrigida; $T_{\text {med }}$ : temperatura medida; $\beta$ : taxa de aquecimento; $\tau$ : tempo de relaxação ou constante de tempo do elemento de medida; $V_{t}$ : volume entre a amostra e detector (DCT) e, $F$ : vazão de gás.

Neste trabalho, todas as temperaturas apresentadas foram corrigidas por esta relação ${ }^{18}$. Todos os perfis de redução foram obtidos em condições livres de limitações de transferência de massa, segundo os critérios utilizados por Monti ${ }^{12}$.

Após alguns experimentos preliminares, escolheram-se os níveis das variáveis para este estudo. As variáveis operacionais e seus níveis encontram-se na Tabela 1.

Tabela 1. Níveis das variáveis no planejamento de experimentos para estudo de TPR

\begin{tabular}{lccll}
\hline Variável & $\begin{array}{c}\text { Nível } \\
\text { inferior }\end{array}$ & $\begin{array}{c}\text { Nível } \\
\text { superior }\end{array}$ & Unidade & Código \\
\hline Taxa de aquecimento & 4 & 8 & ${ }^{\circ} \mathrm{C} \mathrm{min}^{-1}$ & $X_{1}$ \\
Vazão de gás & 30 & 45 & $\mathrm{~mL} \mathrm{~min}^{-1}$ & $X_{2}$ \\
Quantidade de amostra & 350 & 500 & $\mathrm{mmol}^{\prime}$ & $X_{3}$ \\
Concentração de $\mathrm{H}_{2}$ & 3 & 5 & $\%$ & $X_{4}$ \\
\hline
\end{tabular}

Os experimentos de redução termoprogramada foram organizados segundo planejamento fatorial ortogonal conhecido como matriz de Hadamard ${ }^{19}$. Optou-se por um planejamento de quatro variáveis com oito experimentos, o que resulta em uma resolução IV ${ }^{18}$. A matriz de experimentos encontra-se esquematizada na Tabela 2, onde "+" refere-se ao nível superior da variável e "-" ao nível inferior, conforme os intervalos mostrados na Tabela 1. Segundo esta abordagem, é possível a análise das correlações entre as variáveis, além dos efeitos principais, reduzindo significativamente o número de experimentos.

Neste tipo de planejamento fracionado, os resultados obtidos das 4 variáveis principais $\left(X_{1}, X_{2}, X_{3}\right.$ e $\left.X_{4}\right)$ estão sobrepostos com os efeitos das interações triplas, enquanto as interações duplas estão confundidas duas a duas, ou seja, a avaliação destes efeitos deve ser inferida por outros argumentos além dos estatísticos. $A$ priori, as interações de alta ordem como três e quatro variáveis não possuem efeitos significativos sobre os resultados e, por isso, torna-se desnecessário avaliá-las separadamente.

O tamanho dos diâmetros de partícula do catalisador também exerce influência sobre o perfil de redução. Este parâmetro foi

Tabela 2. Planejamento experimental do estudo da influência das variáveis experimentais no perfil de redução termoprogramada

\begin{tabular}{|c|c|c|c|c|c|c|c|}
\hline \multirow[b]{2}{*}{ Experimentos } & \multicolumn{7}{|c|}{ Variáveis e interações } \\
\hline & $\begin{array}{c}X_{2} X_{3} X_{4} \\
X_{1}\end{array}$ & $\begin{array}{c}X_{1} X_{3} X_{4} \\
X_{2}\end{array}$ & $\begin{array}{c}X_{1} X_{2} X_{3} \\
X_{3}\end{array}$ & $\begin{array}{l}X_{3} X_{4} \\
X_{1} X_{2}\end{array}$ & $\begin{array}{l}X_{1} X_{4} \\
X_{2} X_{3}\end{array}$ & $\begin{array}{l}X_{1} X_{2} \\
X_{3} X_{4}\end{array}$ & $\begin{array}{l}X_{2} X_{4} \\
X_{1} X_{3}\end{array}$ \\
\hline 1 & + & + & + & + & + & + & + \\
\hline 2 & + & + & - & + & - & - & - \\
\hline 3 & + & - & + & - & - & - & + \\
\hline 4 & + & - & - & - & + & + & - \\
\hline 5 & - & + & + & - & + & - & - \\
\hline 6 & - & + & - & - & - & + & + \\
\hline 7 & - & - & + & + & - & + & - \\
\hline 8 & - & - & - & + & + & - & + \\
\hline
\end{tabular}


pesquisado para o sistema $\mathrm{CuO} / \mathrm{ZnO} / \mathrm{Al}_{2} \mathrm{O}_{3}$, independentemente do planejamento fatorial, através da seleção de 3 intervalos: 40 a 100; 100 a 250 e 450 a $600 \mu \mathrm{m}$.

\section{RESULTADOS E DISCUSSÃO}

A Figura 1 esquematiza um perfil de redução característico do sistema $\mathrm{CuO} / \mathrm{ZnO} / \mathrm{Al}_{2} \mathrm{O}_{3}$ (razão molar $\mathrm{Cu} / \mathrm{Zn} / \mathrm{Al}=40 / 45 / 15$ ) e as respostas colhidas desta análise. Neste perfil pode-se observar o início do consumo de hidrogênio a, aproximadamente, $130{ }^{\circ} \mathrm{C}$. Este consumo é atribuído à decomposição de espécies superficiais de óxido de cobre finamente dispersas. A seguir, a redução das duas espécies de $\mathrm{Cu}^{2+}\left(195\right.$ e $\left.225^{\circ} \mathrm{C}\right)$ com energias de ativação frente à redução de $61 \pm 9$ e $86 \pm 5 \mathrm{~kJ} \mathrm{~mol}^{-1}$, respectivamente, e um desvio da linha de base até o final da análise, atribuído à decomposição de carbonatos residuais ${ }^{20}$.

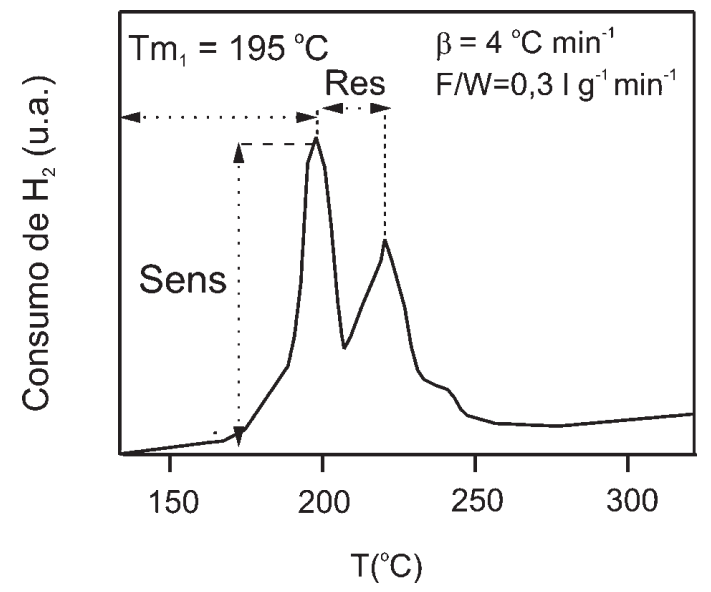

Figura 1. Perfil de redução típico da amostra $\mathrm{CuO} / \mathrm{ZnO} / \mathrm{Al}_{2} \mathrm{O}_{3}(\mathrm{Cu} / \mathrm{Zn} / \mathrm{Al}=$ 40/45/15)

As respostas dos experimentos realizados através do planejamento fatorial encontram-se resumidos na Tabela 3.

Tabela 3. Respostas dos experimentos

\begin{tabular}{lccc}
\hline \multirow{2}{*}{ Experimento } & \multicolumn{3}{c}{ Respostas } \\
\cline { 2 - 4 } & $\mathrm{Tm}_{1}(\mathrm{~K})$ & $\begin{array}{c}\text { Resolução } \\
(\mathrm{min})\end{array}$ & Sensibilidade \\
\hline 1 & 212 & 3,1 & 0,31 \\
2 & 211 & 2,9 & 0,36 \\
3 & 210 & 4,1 & 0,24 \\
4 & 209 & 8,0 & 0,29 \\
5 & 237 & 2,4 & 0,56 \\
6 & 189 & 8,5 & 0,12 \\
7 & 192 & 8,8 & 0,18 \\
8 & 196 & 7,5 & 0,31 \\
\hline
\end{tabular}

A utilização do planejamento estatístico de experimentos em dois níveis possibilita relacionar os efeitos das variáveis com a resposta, através de um polinômio linear:

$\eta=f\left(X_{1}, X_{2}, X_{3}, X_{4}\right)$

onde $\eta$ é a reposta de interesse, $X_{i}$ a variável investigada, normalizada, tendo valor -1 para o seu nível inferior e +1 para o seu nível superior.
Desprezando-se os efeitos de ordem superior a dois (interações três a três), os resultados experimentais podem ser representados pelo polinômio linear:

$$
\eta=b_{0}+\sum_{i=1}^{4} b_{i} X_{i}+\sum_{i=1}^{4} \sum_{\substack{j=1 \\ i \neq j}}^{4} b_{i j} X_{j} X_{i}
$$

onde $b_{0}$ é a média dos resultados experimentais, $b_{i}$ o efeito da variável $X_{i}$, e $b_{i j}$ o efeito da interação de $X_{i} \operatorname{com} X_{j}$. Os coeficientes do polinômio foram obtidos por regressão linear. Utilizando-se apenas os efeitos significativos estatisticamente, o polinômio reduzse, para cada uma das respostas, às Equações:

$$
\begin{aligned}
T m_{1}= & 207,0+10,5 X_{1}+5,0 X_{3}+6,3 X_{4}-9,5 X_{3} X_{4} \\
\text { Res }= & 5,7-2,6 X_{1} \\
\text { Sens }= & 0,30+0,28 X_{1}-0,18 X_{2}+0,09 X_{3}-0,34 X_{4}- \\
& 0,19 X_{1} X_{2}-0,03 X_{2} X_{3}+0,05 X_{1} X_{3}
\end{aligned}
$$

O efeito da taxa de aquecimento $\left(X_{1}\right)$ sobre o deslocamento do perfil de redução, e conseqüentemente da temperatura de máxima redução, é bem conhecido na literatura e utilizado na derivação de parâmetros cinéticos ${ }^{4}$. Nas condições estudadas, esta foi a variável de maior efeito no deslocamento do perfil $\left(T m_{l}\right)$ e na resolução (Res), apresentando importância significativa na sensibilidade (Sens). À medida que a taxa de aquecimento aumenta, o perfil de redução é deslocado para temperaturas mais altas, a resolução diminui e a sensibilidade aumenta. Deste modo, a escolha adequada do valor da taxa de aquecimento para o experimento deve ser um compromisso entre a sensibilidade e a resolução do perfil. Adicionalmente, deve-se levar em conta o intervalo de temperatura no qual a linearidade da programação de temperatura não é comprometida pela potência do forno necessária para atingir altas temperaturas.

É interessante observar que a taxa de aquecimento foi a única variável significativa para a resolução e foi a variável de maior peso sobre a sensibilidade, tendo efeito contrário nas duas respostas. Portanto, para aumentar a sensibilidade da técnica em amostras com múltiplas espécies redutíveis não é recomendável manipular, inicialmente, a taxa de aquecimento, pois isto causará inevitavelmente perda na resolução.

Juntamente com a taxa de aquecimento, a concentração de hidrogênio $\left(X_{4}\right)$ mostrou-se de elevada importância para as respostas analisadas. Com o aumento da concentração de hidrogênio na alimentação, a temperatura de máximo consumo de hidrogênio $\left(T m_{1}\right)$ desloca-se para valores mais baixos. Este efeito pode ser altamente desejável no caso do estudo de espécies que se reduzem a altas temperaturas (ex.: sistema Co/ZMS-5). Além disso, a concentração de hidrogênio define, juntamente com a taxa de aquecimento, as variáveis dominantes para a sensibilidade da técnica. No caso de espécies redutíveis em pequenas quantidades, pode-se utilizar o efeito da concentração de hidrogênio na sensibilidade para facilitar a detecção destas espécies, diminuindo a concentração de agente redutor na corrente de alimentação. Este procedimento deve ser preferido em relação ao aumento da taxa de aquecimento, a qual pode provocar, como mencionado anteriormente, perda de resolução do perfil.

Por outro lado, a utilização de altas concentrações de agente redutor assegura que não haverá consumo excessivo do agente redutor alimentado, o qual pode levar a problemas de transferência de massa e alta pressão parcial de água na amostra, bem como transladar o perfil de redução para temperaturas mais baixas (efeito desejável 
principalmente quando se trabalha com óxidos de difícil redução). Porém, altas concentrações diminuem drasticamente a sensibilidade. Concentrações de 3 a $10 \%$ são normalmente citadas na literatura para redução de sistemas contendo óxido de cobre ${ }^{11,12,14}$.

A influência da vazão de hidrogênio $\left(X_{2}\right)$ foi significativa somente para a sensibilidade, a qual se mostrou dependente de todas as variáveis operacionais. A vazão neste caso determina, junto com a concentração de hidrogênio, o número de mols de hidrogênio alimentados no reator por unidade de tempo. Conseqüentemente, não surpreende que o efeito da vazão de hidrogênio siga a mesma tendência do efeito da concentração de hidrogênio. A utilização de altas vazões deve ser preferida, pois assegura que o consumo de agente redutor não será excessivo, favorece a transferência de calor e a remoção da água formada durante a redução.

$O$ efeito da quantidade de amostra $\left(X_{3}\right)$ redutível foi significativo no deslocamento do perfil de redução, transferindo-o para temperaturas mais altas. Este resultado pode ser por motivos cinéticos ou devido ao aumento da pressão parcial de água no reator que retarda a redução ${ }^{11}$. Neste caso, espera-se que o efeito seja cinético já que as condições de operação asseguraram consumo moderado de hidrogênio e baixa pressão parcial de água (altas vazões).

Ao contrário do esperado, o aumento da quantidade de amostra $\left(X_{3}\right)$ teve uma influência muito pequena na sensibilidade. Para aumentar a sensibilidade, deve-se manipular preferencialmente a concentração do agente redutor na alimentação.

A interação mais forte é a $X_{1} X_{2}+X_{3} X_{4}$. A separação desta interação por meios estatísticos é impossível sem a realização de experimentos adicionais. Mas, por argumentos cinéticos, sabe-se que as variáveis quantidade de espécie redutível $\left(X_{3}\right)$ e concentração de hidrogênio $\left(X_{4}\right)$ interagem entre si significativamente. Desta forma, pode-se inferir que o valor da interação $X_{1} X_{2}+X_{3} X_{4}$ é principalmente devido à interação $X_{3} X_{4}$.

A interação entre a taxa de aquecimento e a quantidade de espécie redutível $\left(X_{I} X_{3}\right)$ já foi detectada anteriormente ${ }^{13}$. Estas variáveis combinadas podem determinar a exaustão de hidrogênio ou o aumento da pressão parcial de água no interior do leito. Porém, neste trabalho, devido à pequena quantidade de espécies redutíveis e às altas vazões de gás, esta interação não foi estatisticamente significativa, ou foi cancelada pela interação $X_{2} X_{4}$, nas respostas posição do perfil $\left(T m_{1}\right)$ e resolução.

O efeito da variação do diâmetro de partícula sobre o perfil de redução foi analisado em 3 intervalos de granulometria, de 40 a $100 ; 100$ a 250 e 450 a $600 \mu \mathrm{m}$. O resultado pode ser visualizado na Figura 2.

Observa-se que com o aumento do diâmetro de partícula há deslocamento da temperatura de máxima taxa de redução para valores mais altos e melhora na resolução entre os picos. Estes efeitos podem ser atribuídos à diminuição da taxa de redução, devido ao aumento da pressão parcial de água nos poros do catalisador à medida que o diâmetro da partícula aumenta. Adicionalmente, é possível observar um acréscimo significativo na área do perfil relativo à decomposição dos carbonatos residuais. Este aumento também pode ser atribuído ao atraso da reação, fazendo com que o consumo de $\mathrm{H}_{2}$ se dê a temperaturas mais altas, mas também pode estar relacionado a um deslocamento da linha de base. Estes efeitos foram previstos por meio de simulação computacional por $\operatorname{Bosh}^{11}$, mas ainda não tinham sido verificados experimentalmente.

\section{CONCLUSÕES}

Nas condições do sistema em estudo e por meio do uso do planejamento estatístico de experimentos, pôde-se constatar que a

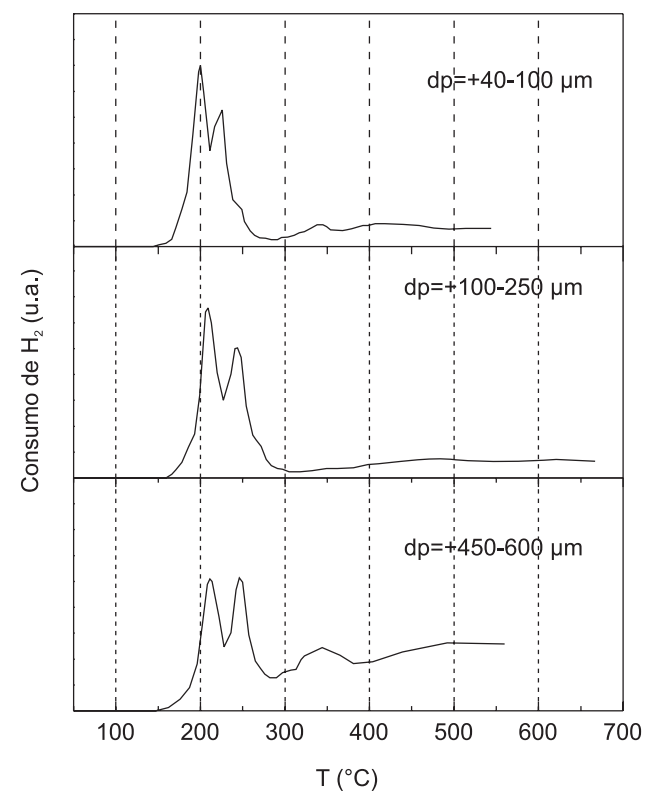

Figura 2. Perfis de redução de amostras com diferentes diâmetros de partícula

técnica de TPR, nos seus aspectos principais, é mais sensível à taxa de aquecimento e à concentração de agente redutor. Portanto, estas são as variáveis que devem ser manipuladas preferencialmente para se obter melhores perfis de TPR. A quantidade de espécies redutíveis e a vazão de gás devem ser tais que possibilitem a minimização dos fenômenos de transporte externo de massa e a exaustão de agente redutor na alimentação facilitando, porém, a transferência de calor. Os diâmetros das partículas devem também ser selecionados para granulometrias menores, de forma a evitar os efeitos difusivos nos perfis de redução.

Com estas informações pode-se ajustar as variáveis operacionais de modo a minimizar problemas de resolução, sensibilidade ou alta temperatura de consumo máximo de hidrogênio. Estes resultados permitem a avaliação crítica das condições experimentais utilizadas, de modo a se obter perfis otimizados e confiáveis em relação à resolução e sensibilidade da técnica.

\section{AGRADECIMENTOS}

M. Nele deseja agradecer ao programa CNPq/PROFIX e E. L. Moreno, ao suporte através da bolsa do CNPq/RHAE.

\section{REFERÊNCIAS}

1. Jones, A.; McNicol, B. D.; Temperature Programmed Reduction for Solid Materials Characterization; Marcel Decker Inc.: New York, 1986.

2. Robertson, S. D.; McNicol, J B. D.; Debaas; H., Kloet; S. C.; Jenkins J. W.; J. Catal. 1975, 37, 424.

3. Cvetanovic, R. J.; Amenomija Y.; Advances in Catalysis 17, Academic Press: New York, apud ref. 1.

4. Gentry, S. J.; Hurst, N. W.; Jones A.; J. Chem. Soc., Faraday Trans.1 1979, $75,1688$.

5. Gentry, S. J.; Walsh, P. T.; J. Chem. Soc. Faraday, Trans. 1 1982, 78, 1515.

6. Isaacs, B. H.; J. Catal. 1982, 77, 43.

7. Delk II, F. S.; Vavere, A.; J. Catal. 1984, 85, 380.

8. Wagstaff, N.; Prins, R.; J. Catal. 1979, 59, 434.

9. Kuznetsova, L. I.; Yurieva, T. M.; Minyukova, T. P.; Ketchik S. V.; Plyasova, L. M.; Boreskov, G. K.; React. Kinet. Catal. Lett. 1982, 19, 355.

10. Malet, P.; Cabalero, A.; J. Chem. Soc., Faraday Trans. 1 1988, 84, 2639.

11. Bosh, H.; Kip, B. J.; Vanommen, J. G.; Gellings, P. J.; J. Chem. Soc., Faraday Trans. 1 1984, 80, 2479.

12. Monti, D. A. M.; Baiker, A.; J. Catal. 1983, 83, 323. 
13. Lin, H.- Y.; Chen, Y. - W.; Li, C.; Thermochim. Acta 2002, 71128, 1.

14. Fierro, G.; Lojacono, M.; Inversi, M.; Porta, P.; Lavecchia, R.; Cioci, F.; J. Catal. 1994, 148, 709.

15. Kislyuk, M. U.; Rozanov, V. V.; Kinet. Catal. 1995, 36, 80.

16. Tonge, K. H.; Thermochim. Acta 1984, 74, 151.

17. Lima, A. A.; Nele, M.; Moreno, E. L.; Andrade, H. M. C.; Appl. Catal., A 1998, 171,31 .
18. Brenner A.; Hucul, D. A.; J. Catal. 1979, 56, 134.

19. Box, W.; Hunter, W. G.; Hunter, J. S.; Statistics for Experimenters: An Introduction to Design, Data Analysis and Model Building, $1^{\text {st }}$ ed., Wiley: New York, 1978.

20. Nele, M.; Dissertação de Mestrado, Universidade Federal da Bahia, Brasil, 1995. 